\title{
Familial clustering of human T lymphotropic virus type 1 uveitis
}

Shinji Araki, Manabu Mochizuki, Kazunari Yamaguchi, Toshiki Watanabe, Ayako Ono, Koichi Yoshimura, Makoto Shirao, Norio Miyata

Human T lymphotropic virus type 1 (HTLV-I) is a retrovirus highly endemic in the Caribbean islands, parts of central Africa and south west Japan. ${ }^{1}$ The virus is a causative agent for a $\mathrm{T}$ cell malignancy (adult $\mathrm{T}$ cell leukaemia/lymphoma, ATL) ${ }^{2}$ and a non-neoplastic inflammatory disorder (HTLV-I associated myelopathy/tropical spastic paraparesis, HAM/TSP). ${ }^{3}$ We have reported that HTLV-I is also a causative agent for a certain type of uveitis. ${ }^{47}$ The familial occurrence of $\mathrm{ATL}^{8}$ and HAM/TSP, ${ }^{9}$ and the prevalence of antibody to HTLV-I in family members ${ }^{10}$ has been reported. Therefore, the familial occurrence of HTLV-I uveitis is also expected, but no such cases have been previously reported. Here we report two familial cases of HTLV-I uveitis.

\section{Case reports}

We have done a clinical survey of uveitis associated with HTLV-I for the past 3 years in south west Japan. In the study, we have found a total of 93 patients with HTLV-I uveitis, ${ }^{7}$ the diagnostic criteria of which are as follows: (1) serum antibody to HTLV-I is positive by particle agglutination assay and enzyme linked immunosorbent assay, and (2) any classic causes for uveitis such as Behçet's disease, sarcoidosis, and the like, are excluded by ophthalmic and systemic examinations. Of the 93 patients with HTLV-I uveitis, we have found two cases of familial occurrence of the disease: a 66-year-old woman and her 62-year-old sister; and a 52-yearold woman and her 26-year-old daughter. All four patients were seropositive for HTLV-I, but did not have ATL and HAM/TSP. The four patients had episodes of floaters and blurred vision with sudden onset. Ophthalmic examinations disclosed that the patients had an inter-

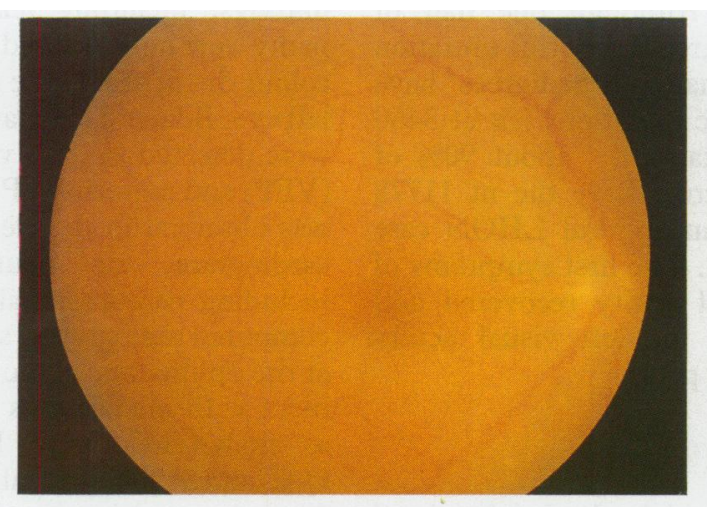

Fig $1 A$

Figure 1 Ophthalmoscopic view of HTLV-I uveitis in a 66-year-old woman $(A)$ and her 62-year-old younger sister $(B)$. The ocular fundus of the two patients was hazy due to a moderate degree of vitreous opacities. The retinal vessels of both patients were slightly dilated. Small snowball-like white exudates were located on the surface of the retinal blood vessels $(B)$. mediate uveitis characterised by moderate inflammatory reaction in the vitreous with fine cells and membranous opacities accompanied by mild iritis and mild retinal vasculitis (Fig 1). The proviral DNA of HTLV-I was detected by polymerase chain reaction from the cells in the anterior chamber of all tested patients. The two families were originally from Kagoshima prefecture, an HTLV-I endemic area and the four patients had no history of blood transfusion. The parents of the patients died many years ago and did not undergo serological tests, although the patients were fed by the breast milk of their mothers. The serum antibody to HTLV-I was negative in the husbands of the sisters, but positive in two children who were fed by the breast milk of the patients. The children did not have uveitis. A serological survey in the family members of the mother and daughter case was not performed because informed consent was hard to obtain.

\section{Comment}

The cases recorded here demonstrate two families of clustering of HTLV-I seropositive idiopathic uveitis. All four patients had an intermediate uveitis with acute onset, the signs and symptoms of which were compatible with classic HTLV-I uveitis reported previously. ${ }^{67}$ HTLV-I is considered to be transmitted through blood transfusion, sexual contact primarily from men to women, and breast milk of HTLV-I infected women. In our study, the HTLV-I carrier state of the patients is most likely transmitted through the breast milk from their mothers based on the following observations: (1) the patients never received a blood transfusion; (2) husbands of the patients were seronegative to HTLV-I, suggesting that sexual contact could be

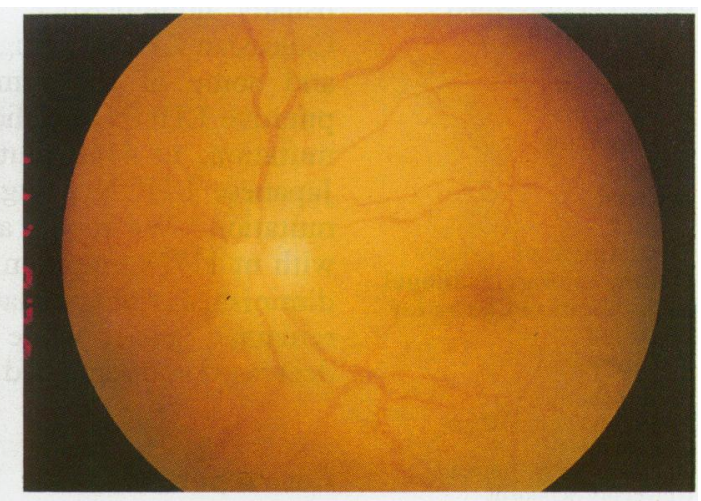

Fig $1 B$ 
excluded; and (3) the patients were breast fed by their mothers although the mothers died many years ago and no serological tests had been performed.

The familial clustering has been reported in a number of autoimmune diseases in which the genetic predisposition plays a role in the disease susceptibility. The present case with familial occurrence of HTLV-I uveitis may suggest an involvement of infectious/autoimmune overlap in the pathogenesis of the disease. The transmission of HTLV-I carrier state and the presence of HTLV-I infected cells in the eye are essential for the development of the disease. However, clinical disease was apparent in only a few of the seropositive family member. The presence of disease only in certain HTLV-I infected family members, therefore, suggests that host factors also play a role in the disease development. It has been reported that immunogenetic factors in the host determine susceptibility to HAM/TSP." Therefore, the survey of immunogenetic factors in HTLV-I uveitis may provide us with further information to understand the pathogenesis of the disease and this is now in progress.

1 Blattner WA. Epidemiology of HTLV-I and associated diseases. In: Blattner WA, ed. Human retrovirology. HTLV. New York: Raven Press, 1990: 251-65.
2 Yoshida M, Seiki M, Yamaguchi K, Takatsuki K. Monoclonal integration of human T-cell leukemia suggests causative role of human T-cell leukemia virus in the disease. Proc Nat Acad Sci USA 1984; 81: 2534-7.

3 Gessain A, Barin F, Vernant JC, Gou O, Calender A, de-The G. Antibody to the human T-lymphotropic virus type-I in patients with tropical spastic paraparesis. Lancet 1985; ii: 407-10.

4 Mochizuki M, Yamaguchi K, Takatsuki K, Watanabe T, Mori S, Tajima K. HTLV-I and uveitis. Lancet 1992; 339. 1110 .

5 Mochizuki M, Watanabe T, Yamaguchi K, Yoshimura K, Nakashima S, Shirao $M$, et al. Uveitis associated with human T-cell lymphotropic virus type I. Am ₹ Ophthalmol 1992; T-cell lymp

6 Mochizuki $M$, Watanabe $T$, Yamaguchi $K$, Takatsuki $K$ Yoshimura K, Shirao M, et al. HTLV-I uveitis: a distinct clinical entity caused by HTLV-I. fpn $\mathcal{f}$ Cancer Res 1992 83: 236-9.

7 Yoshimura K, Mochizuki M, Araki S, Miyata N, Yamaguchi $\mathrm{K}$, Tajima $\mathrm{K}$, et al. Analysis of clinical and immunological features of human T-cell lymphotropic virus type I uveitis. Am $\mathcal{F}$ Ophthalmol 1993 (in press).

8 Yamaguchi K, Yul LS, Shimizu T, Nozawa F, Takeya $M$, Takahashi K, et al. Concurrence of lymphoma type adult

9 Salazar-Grueso EF, Holzer TJ, Gutierrez RA, Casey JM, Desai SM, Devare SG, et al. Familial spastic paraparesis syndrome associated with HTLV-I infection. $N$ Englf Med 1990; 323: 732-7.

10 Miyoshi I, Taguchi H, Fujishima M, Niiya K, Kitagawa T, Ohtsuki Y, et al. Asymptomatic type $\mathrm{C}$ virus carriers in the family of an adult T-cell leukemia patient. Gann 1982; 73: 339-40.

11 Sonoda S. Genetic and immunologic determinants of HTLVI-associated diseases. In: Blattner W, ed. Human retrovirology: HTLV. New York: Raven Press, 1990: $315-26$

\section{An atypical Leber's hereditary optic neuropathy with the 11778 mutation} Yoshihiro Hotta, Mutsuko Hayakawa, Keiko Fujiki, Koutaro Shinohara, Kazushige Sado,
Atsushi Kanai, Kenji Yanashima

Leber's hereditary optic neuropathy (LHON) is a maternally inherited disease that results in optic nerve atrophy, which usually causes permanent low visual acuity. Wallace et $a l^{1}$ reported a $\mathrm{G}$ to $\mathrm{A}$ mutation at nucleotide position (nt) 11778 of mitochondrial DNA (mtDNA) in nine of 11 LHON families. This mutation is usually homoplasmic. Although one half of Caucasian LHON pedigrees carry this mutation and some of the remaining pedigrees have putative LHON specific mutations (ex nt 3460 mutation, nt 4160 mutation), ${ }^{2-4}$ about $90 \%$ of Japanese LHON pedigrees have the nt 11778 mutation. ${ }^{5}$ We report an atypical LHON case with nt 11778 mutation. The first symptoms of diminished right visual acuity recovered and remained good, while the left visual acuity decreased and remained poor.

\section{Case report}

A 32-year-old man complained of right visual loss in February 1990. He was referred in April 1990 after no improvement with medications including corticosteroid at an ophthalmic clinic.
His corrected visual acuities were 20/200 right eye and 20/20 left eye. Automated static perimetry examination showed that the visual field impairment was due to a right co-central scotoma. The temporal aspect of the right optic disc was pale and the left optic disc was slightly reddened. The typical fluorescein angiography findings, including telangiectatic microangiopathy were not observed. Panel D-15 test showed colour disturbance (the axis was not clear) in his left eye. Bilateral elongations of the implicit time were detected in flash visually evoked potential (VEP) and pattern VEP. No remarkable finding was observed in the electroretinogram, electrocardiogram, or neurological examination, including cerebrospinal fluid examination and computed tomography scan. He was followed up at the ophthalmic clinic after no visual improvement with medications including corticosteroid as shown in Figure 1. Vitamins, coenzyme $\mathrm{Q}_{10}$, and ATP were also prescribed. We performed a molecular biological study using peripheral leucocytes for the patient, his father, and his mother (Fig 2). Informed consent was obtained from them. His mother has no clinical 